\title{
A prospective cohort study on radiological and physiological outcomes of recovered COVID-19 patients 6 months after discharge
}

\author{
Mengqi Liu ${ }^{1}$, Fajin $\mathrm{Lv}^{1}$, Yineng Zheng ${ }^{1}$, Kaihu Xiao ${ }^{2,3}$ \\ ${ }^{1}$ Department of Radiology, The First Affiliated Hospital of Chongqing Medical University, Chongqing, China; ${ }^{2}$ Department of Cardiology, \\ Chongqing University Three Gorges Hospital, Chongqing, China; ${ }^{3}$ Department of Cardiology, The Second Affiliated Hospital of Chongqing \\ Medical University, Chongqing, China
}

Contributions: (I) Conception and design: M Liu, F Lv; (II) Administrative support: M Liu, K Xiao; (III) Provision of study materials or patients: M Liu, K Xiao; (IV) Collection and assembly of data: Y Zheng, K Xiao; (V) Data analysis and interpretation: M Liu, F Lv, Y Zheng; (VI) Manuscript writing: All authors; (VII) Final approval of manuscript: All authors.

Correspondence to: Mengqi Liu. Department of Radiology, The First Affiliated Hospital of Chongqing Medical University, Chongqing, China. Email: dany0714@163.com; Kaihu Xiao. Department of Cardiology, Chongqing University Three Gorges Hospital, Chongqing, China.

Email: xiaokh1@163.com.

Background: This study investigated patients' long-term radiological and physiological outcomes with coronavirus disease 2019 (COVID-19).

Methods: A total of 52 patients (26 men and 26 women, 32 with moderate COVID-19 and 20 with severe COVID-19, with a median age of 50.5 years) who had COVID-19 participated in this study. Follow-up thin-section chest computed tomography (CT) scans were performed at 1, 3, and 6 months after discharge. Cardiopulmonary exercise testing was performed on 37 patients 6 months after discharge. The clinical data and the chest CT findings were recorded and analyzed.

Results: The predominant chest CT patterns of abnormalities observed at 6 months after discharge were parenchymal band, interlobular septal thickening, and traction bronchiectasis. The cumulative percentage of the complete radiological resolution was $17 \%, 42 \%, 67 \%$, and $75 \%$ at discharge and 1 , 3, and 6 months after discharge, respectively. A subgroup analysis revealed that $88 \%$ of patients with moderate type and $55 \%$ of patients with severe type COVID-19 achieved complete radiological resolution at 6 months after discharge, and the difference between the 2 groups was significant $(\mathrm{P}<0.001)$. The following risk factors were found to be associated with an incomplete radiological resolution at 6 months after discharge: an age $>50$ years old, the severe type of COVID-19, a hospital stay >18 days, mechanical ventilation, steroid therapy, immunoglobin therapy, an opacity score at discharge $>4$, and a volume of opacity at discharge $>235 \mathrm{~mL}$.

Conclusions: Chest CT lesions were absorbed without any sequelae in most patients with COVID-19; however, fibrotic-like changes and cardiopulmonary insufficiency were still present in a considerable proportion of COVID-19 survivors at 6 months after discharge, especially in patients with severe type COVID-19.

Keywords: Coronavirus infections; computed tomography (CT); viral pneumonia; follow-up

Submitted Nov 23, 2020. Accepted for publication May 13, 2021.

doi: 10.21037/qims-20-1294

View this article at: https://dx.doi.org/10.21037/qims-20-1294 


\section{Introduction}

Coronavirus disease 2019 (COVID-19) has spread throughout the world since late December 2019, leading to a global pandemic. By April 30, 2021, an accumulative 150,110,310 confirmed cases, including 3,158,792 deaths, were reported by the World Health Organization (1). Currently, chest computed tomography (CT) has contributed greatly to the disease assessment of patients with COVID-19. The clinical and radiological characteristics of COVID-19 have been studied extensively. Previous studies have revealed that the typical chest CT manifestations of COVID-19 include peripherally distributed ground-glass opacities (GGO), consolidation, and interlobular septal thickening (2-4). To date, many COVID-19 survivors have recovered and been discharged. COVID-19 survivors have been reported to have common clinical sequelae at 3 months after discharge, including general symptoms, respiratory symptoms, cardiovascular-related symptoms, psychosocial symptoms, and alopecia (5). Recent studies have shown that COVID-19 patients may experience substantial fibrotic consequences (6-11). However, very few studies have undertaken long-term radiological follow-up examinations of discharged patients. This article presents the results of chest CT and cardiopulmonary exercise testing (CPET) in patients with COVID-19 6 months after hospital discharge.

\section{Methods}

\section{Patients}

This prospective cohort study examined COVID-19 survivors who were discharged from Chongqing University Three Gorges Hospital between February 10, 2020, and March 15, 2020. The study was conducted following the Declaration of Helsinki (as revised in 2013). This study was approved by the Ethics Committee of Chongqing University Three Gorges Hospital (No: ChiCTR2000029952). Written informed consent was obtained from each individual participant. The diagnostic and discharge criteria were based on the latest "Pneumonia Diagnosis and Treatment Plan for New Coronavirus Infection" in China (12). Patients were excluded from the study if they met any of the following exclusion criteria: (I) did not undergo a CT scan at admission or discharge; (II) had mild type COVID-19 (i.e., showed no manifestation of pneumonia on chest CT scan); and/or (III) had a history of lung cancer, tuberculosis, or interstitial lung disease.

The clinical data and CT images of each patient were reviewed. COVID-19 severity was classified by reviewing each patient's medical data following Pneumonia Diagnosis and Treatment Plan for New Coronavirus Infection. Specifically, the moderate type was designated when a patient had respiratory symptoms and positive CT findings of pneumonia, while the severe type was designated when a patient met one of the following criteria: (I) had a shortness of breath and a respiratory rate $>30 / \mathrm{min}$; (II) had a saturation of peripheral oxygen $\left(\mathrm{SpO}_{2}\right)<93 \%$ at rest at room temperature; (III) had a partial pressure of oxygen $\left(\mathrm{PaO}_{2}\right)$ /fraction of inspire oxygen $\left(\mathrm{FiO}_{2}\right) \leq 300 \mathrm{mmHg}$; and/ or (IV) had a pulmonary lesion that had progressed more than $50 \%$ in 24 to 48 hours based on radiological imaging.

\section{Computed tomography protocols}

All chest CT examinations were performed using one of the following 2 multidetector CT scanners: (I) Siemens Somatom Sensation (Siemens, Erlangen, Germany); or (II) uCT 510 (United Imaging, China). All patients underwent non-contrast-enhanced scanning in the supine position. The imaging parameters were as follows: a tube voltage of $120 \mathrm{kV}$; a tube current of $150 \mathrm{~mA}$; and a scanning slice thickness of $5 \mathrm{~mm}$. The images were reconstructed with a slice thickness of $1.0 \mathrm{~mm}$ and an interval of $1.0 \mathrm{~mm}$. The scanning range included the whole chest from the first ribs to the diaphragm.

Chest thin-section CT scans were regularly performed at definite time points for each patient. We reviewed $5 \mathrm{CT}$ scans comprising the initial CT scan at admission, the CT scan at discharge, and the CT scans taken at examinations at 1,3 , and 6 months after discharge.

\section{Computed tomography image analysis}

Two thoracic radiologists (with 8 years and 25 years of experience, respectively) blinded to the clinical data interpreted the CT scans independently. Any disagreement was resolved by discussion to reach a consensus. The initial and follow-up CT scans of each patient were evaluated and compared for the presence and distribution of the following abnormalities: (I) pure GGO; (II) consolidation; (III) air bronchogram; (IV) crazy paving pattern; (V) nodules; (VI) interlobular septal thickening; (VII) irregular interfaces; (VIII) coarse reticular pattern; (IX) traction bronchiectasis; (X) parenchymal bands; (XI) pleural effusion; (XII) pericardial effusion; and/or (XIII) lymphadenopathy, which was defined as a lymph node $>1 \mathrm{~cm}$ in short-axis diameter. 

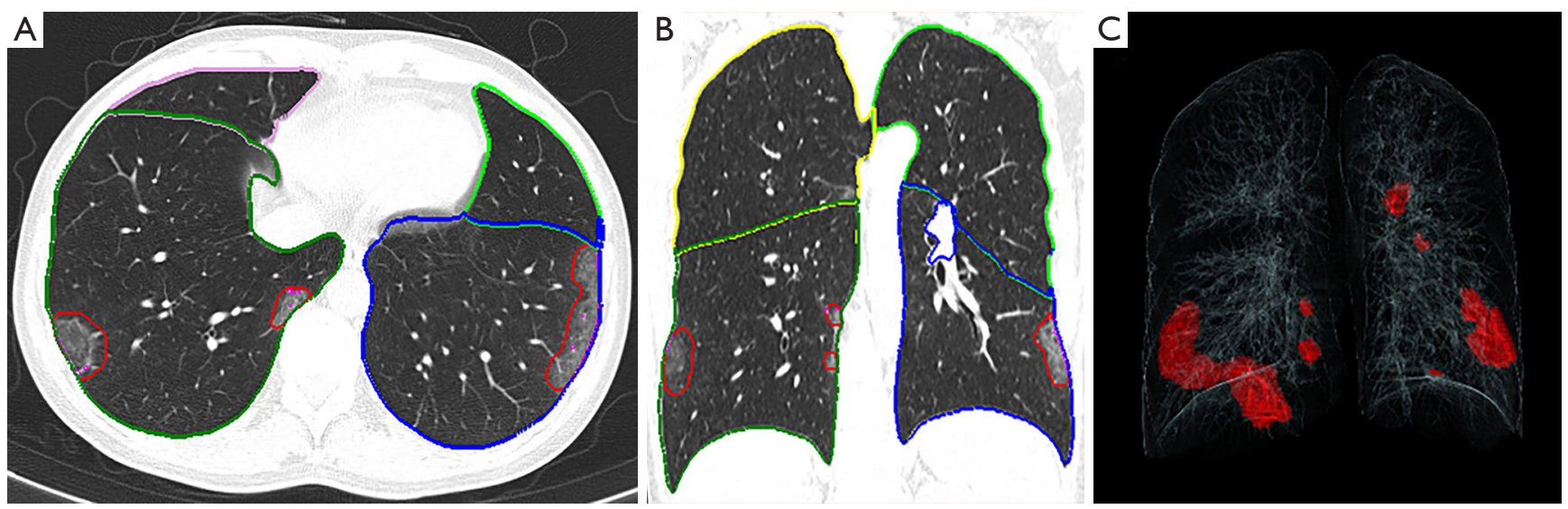

Figure 1 Representative postprocessing results of chest CT scans performed with siemens CT pneumonia analysis. A 42 -year-old male complaining of fever and cough for 1 day (moderate type of COVID-19, Opacity score =2, volume of opacity =89.0 mL). (A) Axis plane of chest CT. (B) Coronal plane of chest CT showed bilateral ground-glass opacities with subpleural distribution. (C) 3D-visualization of CT volume rendering technique showed the diffuse extent of the opacities.

All the above terms were defined following the Fleischner Society's guidelines (13). In addition, the outer one-third of the lung was defined as peripheral, and the rest was defined as central. Further, an artificial intelligence system (CT Pneumonia Analysis, Siemens Healthineers, Siemens, Erlangen, Germany) was used to detect and quantify the volume of the opacities (including GGO and consolidative lesions) automatically (see Figure 1).

The extent of pneumonia was segmented automatically and checked by an experienced radiologist, and the contours of the lesions were revised manually on the condition of inaccurate automatic segmentation. The following 4 parameters were computed for each CT scan: the opacity score; the lung volume; the volume of opacity; and the percentage of opacity (the ratio of the volume of opacity to lung volume). Each of the 5 lung lobes was scored automatically on a scale of 0 to 4 (on which 0 indicated $\leq 1 \%$ involvement, 1 indicated $2-25 \%$ involvement, 2 indicated $26-50 \%$ involvement, 3 indicated $51-75 \%$ involvement, and 4 indicated $>75 \%$ involvement). The total opacity score was the sum of the 5 lobar scores and ranged from 0 to 20 . The above quantitative parameters calculated by the artificial intelligence software and radiological features of the CT images at admission were regarded as the baseline, and the indicators of the follow-up CT scans were compared. Additionally, a complete radiological resolution was defined as the absence of any chest CT abnormality related to infection (14), and the cumulative percentage of complete radiological resolution at each follow-up was calculated.

\section{Cardiopulmonary exercise testing}

A resting lung function test was performed immediately before performing CPET. Forced expiratory volume in 1 second (FEV1) and forced vital capacity (FVC) were measured using a QUARK CPET system (Cosmed, Rome, Italy). After that, CPET was performed after a 20 -minute rest. The incremental exercise was done on a treadmill (Cosmed, Rome, Italy). The workload was selected according to the patient's height, weight, and daily activity capacity. Patients were asked to exert their maximum effort. During exercise, blood pressure, $\mathrm{SPO}_{2}$, and electrocardiography (ECG) were closely monitored. If a patient experienced difficulty breathing, chest tightness, or if the ECG showed an ST-segment and T-wave change indicating myocardial hypoxia, or if the patient could not tolerate the exercise, the workload was considered to have reached the maximum limit. The following parameters were measured and recorded subsequently: peak oxygen uptake $\left(\mathrm{VO}_{2}\right)$, minute ventilation (VE), carbon dioxide production $\left(\mathrm{VCO}_{2}\right), \mathrm{VO}_{2}$ at anaerobic threshold (AT), peak metabolic equations (METs), and $\mathrm{VE} / \mathrm{VCO}_{2}$ slope. The peak $\mathrm{VO}_{2}$ was defined as the highest average $\mathrm{VO}_{2}$ for a given 20-second interval within the last 60 seconds of exercise. The VE/ $\mathrm{VCO}_{2}$ slope was determined with the linear regression slope of $\mathrm{VE}$ and $\mathrm{VCO}_{2}$ from the first second of the exercise period until the respiratory compensation point. The AT was defined as the $\mathrm{VO}_{2}$ level where the $\mathrm{VE} / \mathrm{VCO}_{2}$ decreased or remained constant while the $\mathrm{VE} / \mathrm{VO}_{2}$ persistently increased. 


\section{Statistical analysis}

All statistical analyses were performed using SPSS (version 17.0, IBM Corp., Armonk, NY, USA). A single-sample Kolmogorov-Smirnov analysis was used to test the variance in homogeneity. The continuous variables (e.g., age, lung volume, and FVC) are expressed as mean \pm standard deviation (SD) or median [interquartile range (IQR)] and compared using a 2 independent samples $t$-test (homogeneity of variance) or a Mann-Whitney $U$ test (heterogeneity of variance). The categorical variables (e.g., comorbidity, symptoms, and CT signs) were expressed as numbers (\%) and compared by the $\chi^{2}$ test or Fisher's exact test between groups. A $\mathrm{P}<0.05$ was considered statistically significant.

\section{Results}

\section{Clinical characteristics of patients}

A total of 52 COVID-19 survivors (26 male and 26 female) participated in the study. Of these patients, 32 were diagnosed with moderate COVID-19, and 20 were diagnosed with severe COVID-19. Patients' median age was 50.5 (IQR: 41.3-57.0) years old. The median days from symptom onset to admission were 6.0 (IQR: 3.3-7.0) days. The most prevalent initial symptoms were fever (73\%) and cough (63\%). Less common symptoms were sputum, dyspnea, diarrhea, headache, chest pain, and sore throat. Hypertension (13\%) and diabetes (12\%) were the most common concomitant diseases. A steroid hormone was given to $12(23 \%)$ patients, while 10 and 7 patients received immunoglobins and mechanical ventilation, respectively. The median hospital stay was 17.0 (IQR: 13.0-23.8) days. A subgroup analysis revealed that patients in the severe group were older (median age, 56.5; IQR: 49.5-71.3) than those in the moderate group (median age, 47.0; IQR: 35.3-57.9) $(\mathrm{P}=0.004)$. There were no significant differences in the clinical symptoms and the proportion of patients with comorbidities between the 2 groups. However, compared with the moderate group, more patients in the severe group received mechanical ventilation, and more patients were treated with corticosteroids and immunoglobins $(\mathrm{P}<0.001)$. The detailed clinical characteristics of the patients are summarized in Table 1.

\section{Dynamic chest computed tomography evaluations}

We assessed 5 CT scans for each patient. The median interval between disease onset and the initial chest CT scan was 6.0 (IQR: 3.3-7.0) days, and the median intervals of the moderate and severe groups did not differ statistically $(\mathrm{P}=0.117)$. The detailed dynamic changes of the chest $\mathrm{CT}$ characteristics are listed in Table 2 and shown in Figures 2 and 3. As summarized in Table 2, the predominant CT manifestations at admission included GGO (90\%), consolidation (44\%), and interlobular septal thickening (44\%). The less common CT features included parenchymal band $(38 \%)$, irregular interface $(37 \%)$, coarse reticular pattern (31\%), crazy paving pattern (29\%), air bronchogram (19\%), and nodules (12\%). The rare CT features included pericardial effusion (10\%), pleural effusion $(4 \%)$, and lymphadenopathy $(2 \%)$. The quantitative parameters calculated by the CT pneumonia analysis software revealed that the median opacity score was 4.8 , the median volume of opacity was $381 \mathrm{~mL}$, and the median percentage of opacity was $11.9 \%$ at admission. At discharge, the positive count of GGO, crazy paving pattern, and air bronchogram decreased, while the positive count of the following fibrotic changes increased: interlobular septal thickening, irregular interface, parenchymal band, and traction bronchiectasis. After discharge, all these radiological signs showed various degrees of resolution (see Figures 4,5), and all of the quantitative parameters of the opacities (i.e., the opacity score, the volume of opacity, and the percentage of opacity) gradually decreased with time. However, the following lesions did not fully resolve until 6 months after discharge: GGO (8\%), consolidation (4\%), interlobular septal thickening (25\%), irregular interface (12\%), coarse reticular pattern (12\%), the parenchymal band (25\%), and traction bronchiectasis (25\%). Fibrotic changes (parenchymal bands, reticular pattern, and traction bronchiectasis) were present in 16 patients $(31 \%)$ at discharge, and fibrotic lesions were still present in 13 of these 16 patients at the 6-month follow-up examination. Notably, no patient developed fibrotic changes during the follow-up period.

The cumulative percentage of a complete radiological resolution was calculated at each follow-up examination. Only $9(17 \%)$ patients achieved complete radiological resolution at discharge. After that, the cumulative percentage of a complete radiological resolution was $42 \%$ (22 patients), 67\% [35], and 75\% [39] at 1, 3, and 6 months after discharge, respectively. As Figure 3 and Table 2 show, $8(25 \%)$ patients in the moderate group achieved complete radiological resolution at discharge, but only $1(1 \%)$ patient in the severe group achieved complete resolution at the time. At 1 month after discharge, the cumulative percentage of a complete radiological resolution increased to $56 \%$ for 
Table 1 Demographic and clinical characteristics of the 52 patients with COVID-19

\begin{tabular}{|c|c|c|c|c|}
\hline Characteristics & Total $(\mathrm{N}=52)$ & Moderate group $(\mathrm{N}=32)$ & Severe group $(\mathrm{N}=20)$ & $P$ value \\
\hline Female & $26(50 \%)$ & $17(53 \%)$ & $9(45 \%)$ & \\
\hline Age (years) & $50.5(41.3-57.0)$ & $47.0(35.3-57.9)$ & $56.5(49.5-71.3)$ & 0.004 \\
\hline Diabetes & $6(12 \%)$ & $2(6 \%)$ & $4(20 \%)$ & 0.131 \\
\hline Hypertension & $7(13 \%)$ & $5(16 \%)$ & $2(10 \%)$ & 0.563 \\
\hline Chronic obstructive pulmonary disease & $2(4 \%)$ & $0(0 \%)$ & $2(10 \%)$ & 0.068 \\
\hline Cardiovascular disease & $2(4 \%)$ & $1(3 \%)$ & $1(5 \%)$ & 0.732 \\
\hline Fever & $38(73 \%)$ & $22(69 \%)$ & $16(80 \%)$ & 0.374 \\
\hline Cough & $33(63 \%)$ & $18(56 \%)$ & $14(70 \%)$ & 0.321 \\
\hline Sputum & $12(23 \%)$ & $7(22 \%)$ & $5(25 \%)$ & 0.795 \\
\hline Dyspnea & $9(17 \%)$ & $3(9 \%)$ & $6(30 \%)$ & 0.056 \\
\hline Headache & $10(19 \%)$ & $5(16 \%)$ & $5(25 \%)$ & 0.404 \\
\hline Weakness of limbs & $14(27 \%)$ & $7(22 \%)$ & 7 (35\%) & 0.299 \\
\hline Chest pain & $8(15 \%)$ & $5(16 \%)$ & $3(15 \%)$ & 0.952 \\
\hline Days from onset to admission & $6.0(3.3-7.0)$ & $4.0(3.0-7.0)$ & $6.5(6.0-7.0)$ & 0.117 \\
\hline Hospital stay (day) & $17.0(13.0-23.8)$ & $16.0(12.3-22.5)$ & $18.0(13.0-25.0)$ & 0.631 \\
\hline
\end{tabular}

Data are median (interquartile range) or $\mathrm{n}(\%)$.

the moderate group and $20 \%$ for the severe group; the difference between the 2 groups was significant $(\mathrm{P}=0.010)$. Similarly, up to $28(88 \%)$ patients in the moderate group achieved complete radiological resolution at 6 months after discharge, but only $11(55 \%)$ patients in the severe group achieved complete radiological resolution; the difference between the 2 groups was significant $(\mathrm{P}<0.001)$.

\section{Factors associated with resolution}

Of the patients, 39 (75\%) achieved complete radiological resolution at 6 months after discharge; however, 13 patients still had a certain number of residual pulmonary lesions. A Chi-square test was performed to explore which factors correlated with radiological resolution. There was no obvious difference in complete radiological resolution at the 6-month follow-up examination between males and females. However, the following were found to be risk factors associated with incomplete radiological resolution: an age $>50$ years old, severe COVID-19, a hospital stay >18 days, mechanical ventilation, steroid therapy, immunoglobin therapy, an opacity score at discharge $>4$, and a volume of 


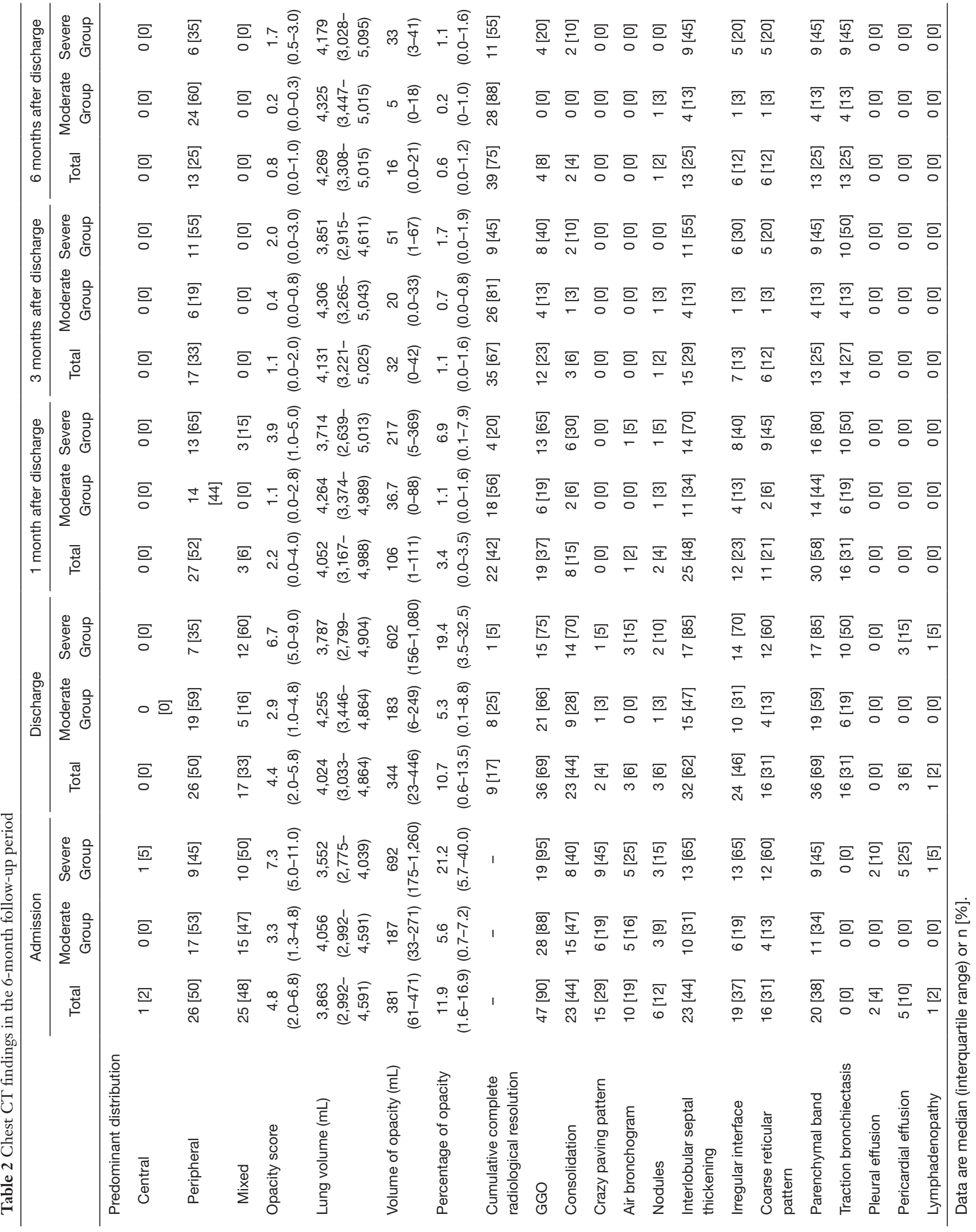




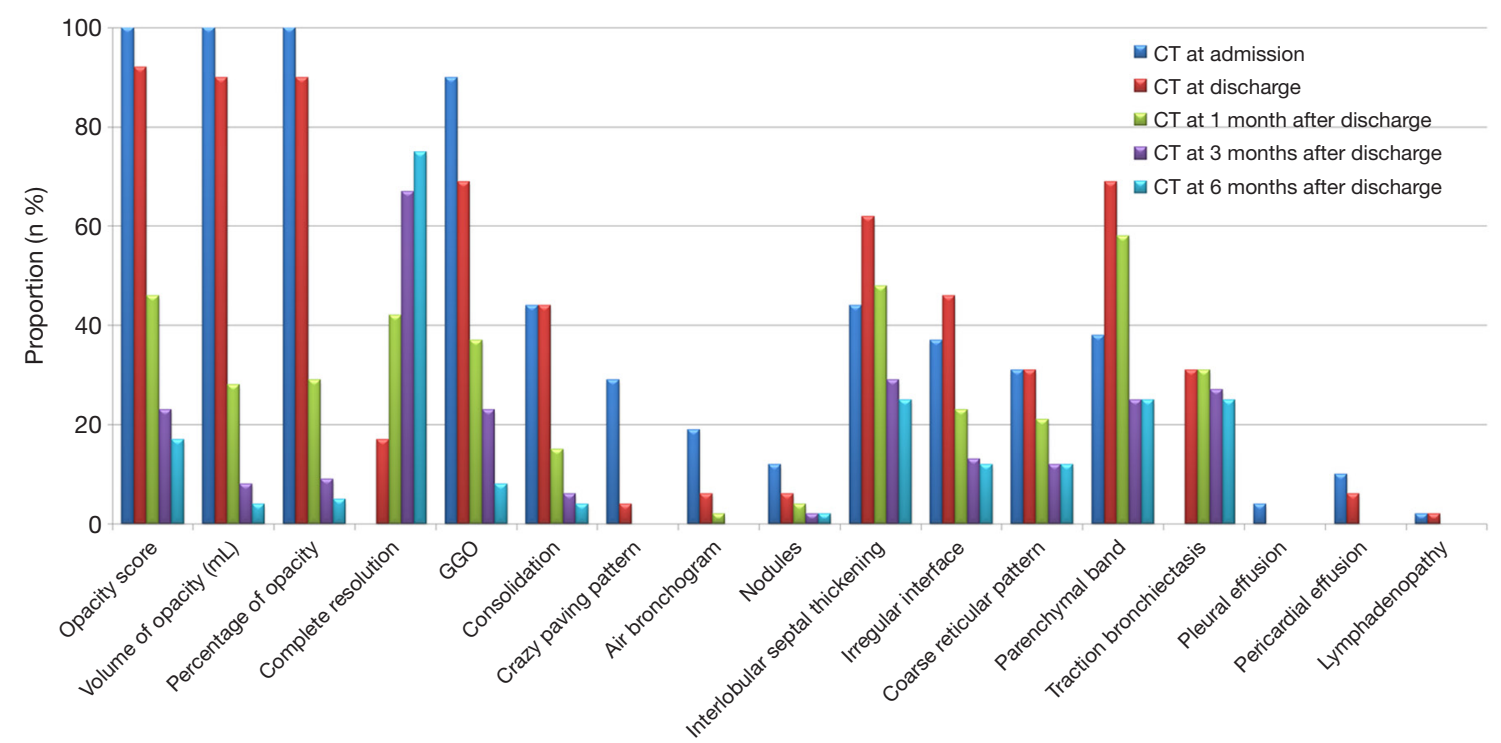

Figure 2 Proportion of positive patients with typical imaging features of COVID-19 pneumonia on chest CT at different time points. Opacity score, the volume of opacity, and the percentage of opacity (the ratio of volume of opacity to lung volume) measured on the chest CT scan at admission were regarded as baseline (100\%). GGO, ground-glass opacities.
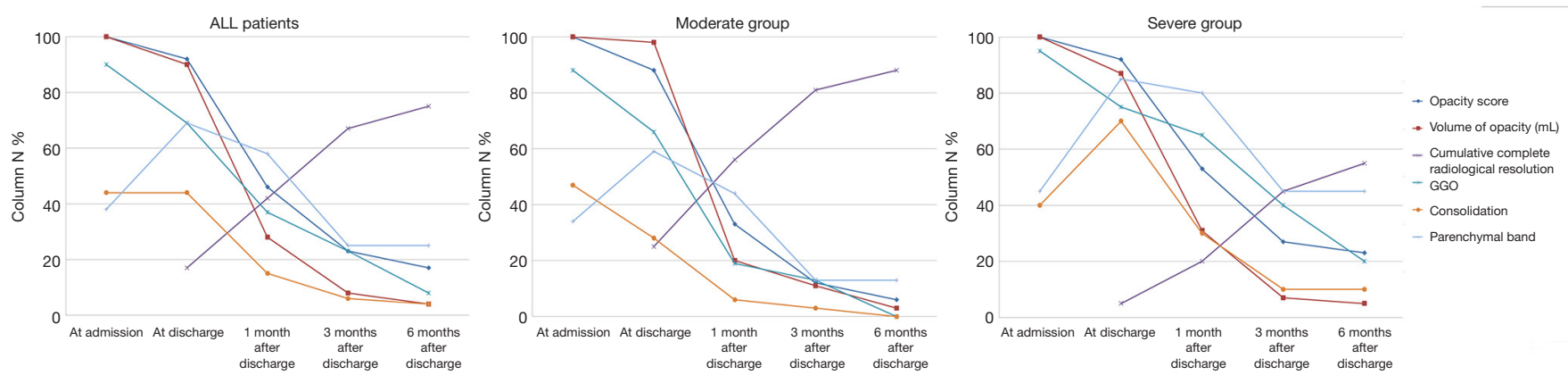

Figure 3 Dynamic changes of chest CT manifestation at different time points. The abnormalities in the lungs were absorbed gradually after discharge, and the moderate group was found to have a faster rate of complete radiological resolution than the severe group.

opacity at discharge $>235 \mathrm{~mL}$ (see Table 3).

\section{Cardiopulmonary exercise testing}

Resting lung function tests and CPET were performed on 37 of the 52 patients at 6 months after discharge. The peak $\mathrm{VO}_{2}$ was normal $(>20 \mathrm{~mL} / \mathrm{kg} / \mathrm{min})$ in 17 patients $(46 \% ; 13$ with moderate COVID-19 and 4 with severe COVID-19), the peak $\mathrm{VO}_{2}$ was $16-20 \mathrm{~mL} / \mathrm{kg} / \mathrm{min}$ in 11 patients $(30 \% ; 7$ with moderate COVID-19 and 4 with severe COVID-19), and the peak $\mathrm{VO}_{2}$ was $10-16 \mathrm{~mL} / \mathrm{kg} / \mathrm{min}$ in 9 patients $(24 \%$; 4 with moderate COVID-19 and 5 with severe COVID-19). A total of 30 patients $(81 \%)$ had normal $\mathrm{VO}_{2}$ at AT
(>14 $\mathrm{mL} / \mathrm{kg} / \mathrm{min}$ ), and the remaining 7 patients $(19 \%)$ had a $\mathrm{VO}_{2}$ at $\mathrm{AT}$ of $11-14 \mathrm{~mL} / \mathrm{kg} / \mathrm{min}$ (3 with moderate COVID-19 and 4 with severe COVID-19). However, there were no apparent differences in FEV1, FVC, $\mathrm{VO}_{2}, \mathrm{VO}_{2}$ at AT, METs, and VE/VCO2 between the moderate and severe groups. The details are summarized in Table 4.

\section{Discussion}

Severe acute respiratory syndrome coronavirus 2 (SARSCoV-2) is the third-largest coronavirus outbreak, after severe acute respiratory syndrome (SARS) and the Middle East respiratory syndrome (MERS). After the SARS 

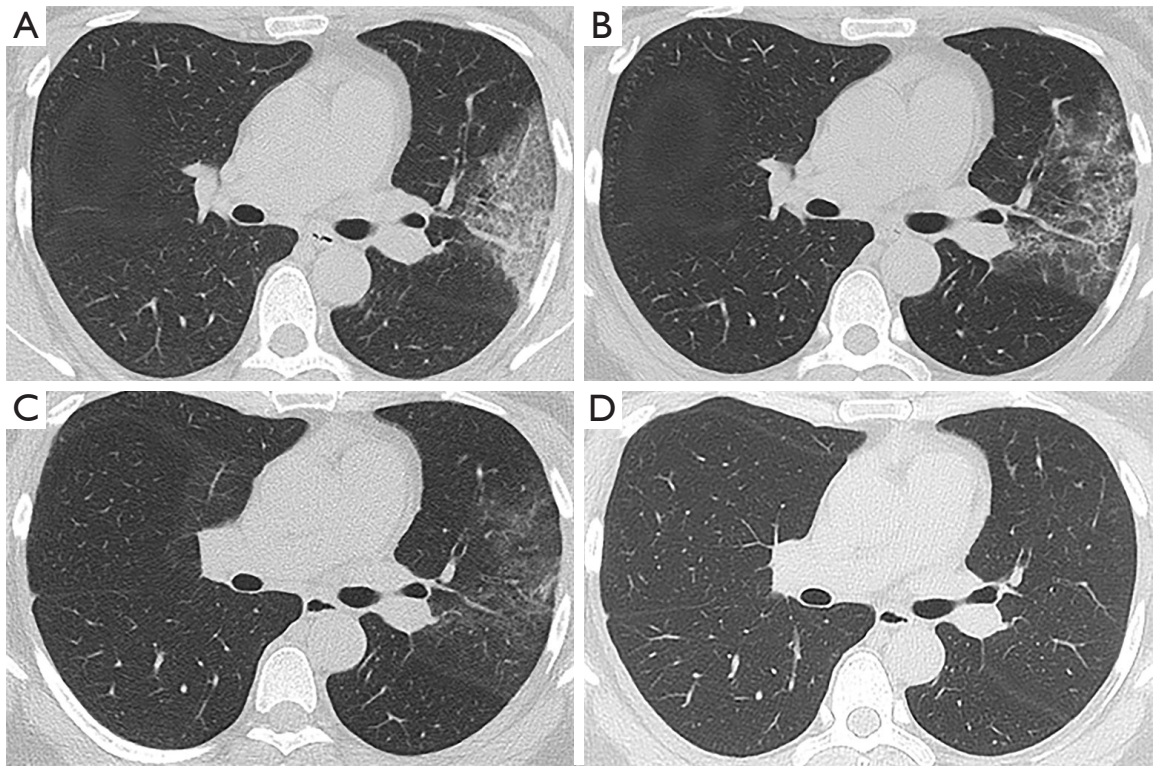

Figure 4 Follow-up chest CT scans of a 39-year-old female with moderate COVID-19. (A) At admission, CT scan showed ground-glass opacities, consolidation, and interstitial thickening in left upper lobe; (B) at discharge (13 days after admission), most consolidative lesions were absorbed; (C) at 1 month after discharge, most lesions were resolved and parenchymal bands with residual ground-glass opacities were observed; (D) at 3 months after discharge, complete radiological resolution was achieved.
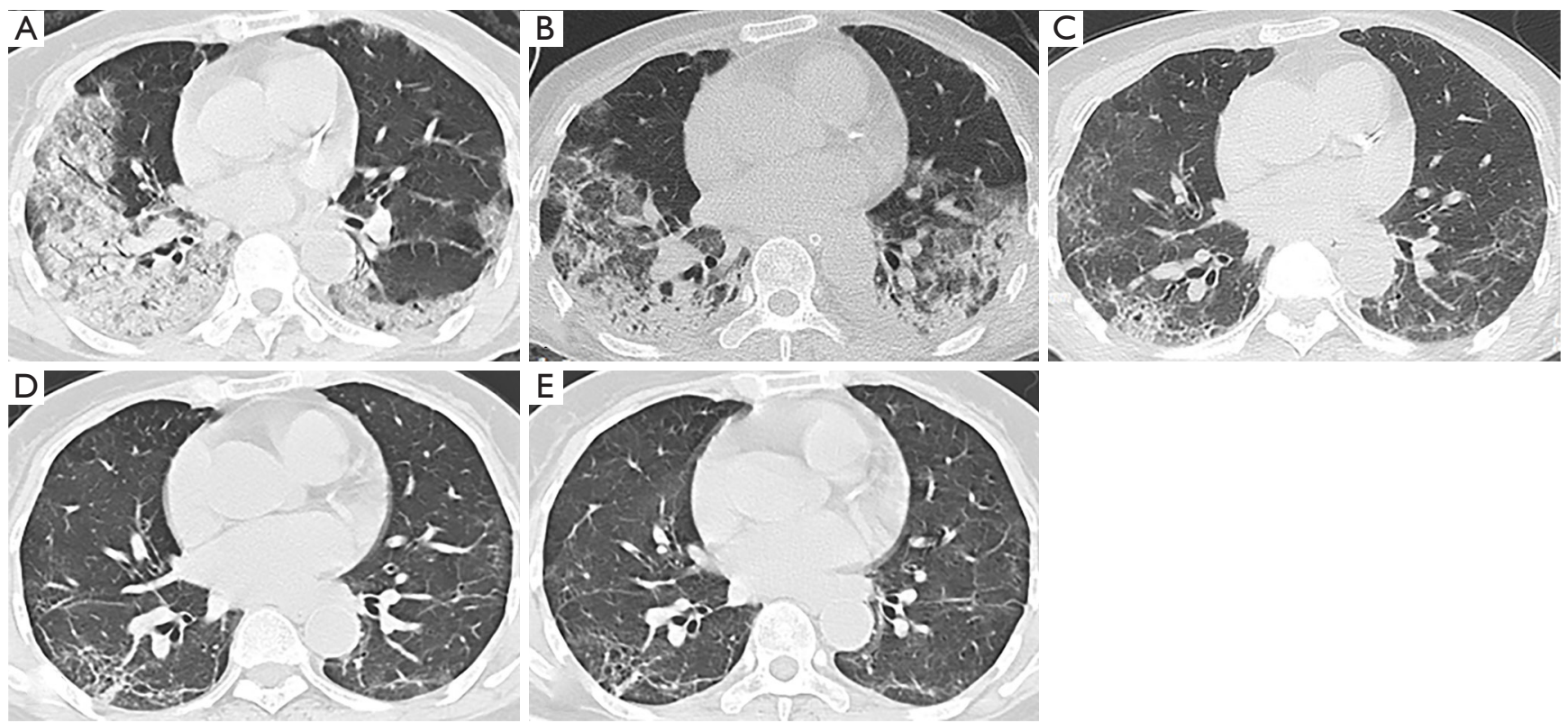

Figure 5 Follow-up chest CT scans of a 74-year-old female with severe COVID-19. (A) At admission, CT scan showed groundglass opacities, consolidation, and air bronchogram in bilateral lower lobes; (B) at discharge (24 days after admission), CT scan showed consolidation with decreased lesion extent in the right lung and increased lesion extent in the left lung; (C) at 1 month after discharge, most consolidative lesions were resolved and interstitial thickening with residual ground-glass opacities were observed; (D) at 3 months after discharge, CT scan showed parenchymal bands, irregular interfaces, and traction bronchiectasis; (E) at 6 months after discharge, the lesions on the last follow-up CT were still present. 
Table 3 Correlative factors with radiological resolution

\begin{tabular}{|c|c|c|c|}
\hline & \multicolumn{2}{|c|}{ Complete radiological resolution } & \multirow{2}{*}{$P$ value } \\
\hline & Yes & No & \\
\hline \multicolumn{3}{|l|}{ Age (years) } & 0.004 \\
\hline$>50$ & 15 & 11 & \\
\hline$\leq 50$ & 24 & 2 & \\
\hline \multicolumn{3}{|l|}{ Severity } & 0.008 \\
\hline Severe group & 11 & 9 & \\
\hline Moderate group & 28 & 4 & \\
\hline \multicolumn{3}{|l|}{ Sex } & 0.749 \\
\hline Male & 19 & 7 & \\
\hline Female & 20 & 6 & \\
\hline \multicolumn{3}{|l|}{ Hospital stay (day) } & 0.006 \\
\hline$>18$ & 13 & 10 & \\
\hline$\leq 18$ & 26 & 3 & \\
\hline \multicolumn{3}{|c|}{ Mechanical ventilation } & 0.002 \\
\hline Yes & 2 & 5 & \\
\hline No & 37 & 8 & \\
\hline \multicolumn{3}{|l|}{ Steroid therapy } & 0.002 \\
\hline Yes & 5 & 7 & \\
\hline No & 34 & 6 & \\
\hline \multicolumn{3}{|c|}{ Immunoglobin therapy } & 0.004 \\
\hline Yes & 4 & 7 & \\
\hline No & 35 & 7 & \\
\hline \multicolumn{3}{|c|}{ Opacity score at discharge } & $<0.001$ \\
\hline$>4$ & 12 & 12 & \\
\hline$\leq 4$ & 27 & 1 & \\
\hline \multicolumn{3}{|c|}{ Volume of opacity $(\mathrm{mL})$ at discharge } & $<0.001$ \\
\hline$>235$ & 11 & 11 & \\
\hline$\leq 235$ & 28 & 2 & \\
\hline
\end{tabular}

outbreak, many patients recovered; however, lung function and radiological abnormalities were observed in $75.4 \%$ of patients who recovered from SARS at 6-month follow-up examinations (15). Thus, this raises an important question for doctors: Will patients who have recovered from COVID-19 have any clinical sequelae? To date, emerging studies have described the sequelae of COVID-19 survivors $(5-7,9,16,17)$; however, the long-term outcomes in both pulmonary function and chest CT imaging remain unknown. In the present study, we examined the results of CPET, and dynamic changes of chest CT scans in patients with COVID-19 6 months after they had been discharged from the hospital. Notably, we found that patients' chest CT abnormalities were still obvious at discharge. Compared to the abnormalities found on the CT scans at admission, the abnormalities in the lungs at discharge had been gradually absorbed. The cases with GGO, crazy paving pattern, and air bronchogram were reduced from $90 \%, 29 \%$, and $19 \%$, to $69 \%, 4 \%$, and $6 \%$ of cases, respectively. However, the cases with an irregular interface, parenchymal band, and traction bronchiectasis were increased, which implied that pulmonary fibrotic changes increased significantly.

As reported previously, pulmonary fibrosis may develop early in patients who have recovered from SARS (18), and fibrosis lesions may persist (15). Conversely, in our cohort, we observed pulmonary fibrotic changes were absorbed with time, which supports the findings of previous studies $(6,19)$. The reversibility of these lesions probably indicates that the radiological fibrotic changes did not signify actual pathologic fibrosis (20). Thus, the question of whether such lesions will resolve requires further research. In the present study, 22 (42\%) patients achieved complete radiological resolution at the 1-month follow-up examination.

Similarly, Liu et al. reported that the pulmonary abnormalities of $53.0 \%$ of COVID-19 survivors were fully absorbed 3 weeks after discharge (17). Liu et al.'s study included a smaller proportion of severe cases than our study $(4.7 \%$ vs. $38 \%)$, which may be why the radiological resolution rate in our study was relatively slower. At 6 months after discharge, the median volume of opacity was reduced to $16 \mathrm{~mL}$, and the cumulative complete radiological resolution rate reached $75 \%$. Our findings showed that pulmonary damage caused by COVID-19 might repair without any sequelae.

We compared the dynamic changes in chest CT scans between patients in the moderate and severe groups. Patients in the moderate group achieved a faster rate of complete radiological resolution than those in the severe group. At 6 months after discharge, up to 28 (88\%) patients in the moderate group achieved complete radiological resolution, compared to only 11 (55\%) patients in the severe group. In addition, GGO and consolidation were fully absorbed in all the patients in the moderate group, but up to $20 \%$ and $10 \%$ of the severe group demonstrated residual GGO and consolidation, respectively. Similarly, compared to the moderate group, more patients in the 
Table 4 Results of spirometry and cardiopulmonary exercise testing at 6 months after discharge

\begin{tabular}{|c|c|c|c|c|}
\hline & All patients $(\mathrm{N}=37)$ & Moderate group $(\mathrm{N}=24)$ & Severe group $(\mathrm{N}=13)$ & $P$ value \\
\hline Peak VO ${ }_{2}(\mathrm{~mL} / \mathrm{min} / \mathrm{kg})$ & $19.1 \pm 4.1$ & $20.0 \pm 3.8$ & $17.5 \pm 4.5$ & 0.087 \\
\hline $\mathrm{VO}_{2}$ at $\mathrm{AT}(\mathrm{mL} / \mathrm{min} / \mathrm{kg})$ & $15.8 \pm 3.5$ & $16.4 \pm 3.3$ & $14.6 \pm 3.5$ & 0.156 \\
\hline METs & $5.5 \pm 1.2$ & $5.7 \pm 1.1$ & $5.1 \pm 1.3$ & 0.082 \\
\hline
\end{tabular}

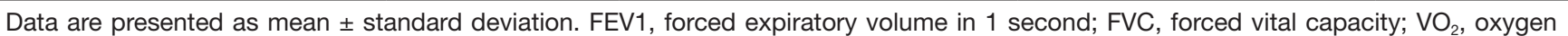
consumption; AT, anaerobic threshold; METs, metabolic equations; $\mathrm{VE} / \mathrm{VCO}_{2}$, ventilatory equivalent for carbon dioxide.

severe group demonstrated residual fibrotic-like changes. Thus, chest CT abnormalities appear to be absorbed without any sequelae in most patients with the moderate type of COVID-19; however, fibrotic changes may still be present in a considerable proportion of patients with severe COVID-19 survivors 6 months after discharge.

We investigated the correlative factors with radiological resolution. A previous study reported that male sex was a high-risk factor for worse outcomes in patients with COVID-19 (21). However, in the present study, there was no significant difference concerning complete radiological resolution at the 6-month follow-up examination between males and females, which is consistent with the findings of several recent studies $(7,8,17)$. Additionally, similar to previous studies $(8,17,22)$, we found that older age was a risk factor for poor radiological resolution. We also found other factors associated with the radiological resolution, including a hospital stay $>18$ days, mechanical ventilation, steroid therapy, immunoglobin therapy, an opacity score at discharge $>4$, and a volume of opacity at discharge $>235 \mathrm{~mL}$; all of which may be related to the severity of the disease. Thus, COVID-19 survivors of older age or those with severe conditions are more likely to present with residual reserve lesions on chest CT scans.

CPET was performed on 37 patients 6 months after discharge. Lung function abnormalities were observed in $25 \%$ of COVID-19 survivors at the 3 -month follow-up examination (22). CPET provides integrated data about a patient's cardiovascular, ventilatory, and metabolic response to physical effort (23). Thus, CPET can provide more physiological information than a lung function test. In the present study, the $\mathrm{VO}_{2}$ at AT was normal $(>14 \mathrm{~mL} / \mathrm{kg} / \mathrm{min})$ in 30 patients $(81 \%)$, and the $\mathrm{VO}_{2}$ at AT was $11-14 \mathrm{~mL} / \mathrm{kg} / \mathrm{min}$ in the remaining 7 patients (19\%), which implied mild-tomoderate impairment in aerobic capacity based on Weber's classification (24). However, abnormal peak $\mathrm{VO}_{2}$ occurred in up to $54 \%$ of patients. A recent study showed that $63 \%$ of patients experienced symptoms of fatigue or muscle weakness 6 months after discharge (25). In our study, most patients with abnormal peak $\mathrm{VO}_{2}$ stopped the test because of lower-extremity muscle fatigue rather than chest tightness or shortness of breath. Thus, the $\mathrm{VO}_{2}$ results at AT might more reliably reflect cardiopulmonary function than that of peak $\mathrm{VO}_{2}$.

We had no baseline CPET results for patients. Given that patients' pre-COVID data were not available, it cannot be concluded that the lower $\mathrm{VO}_{2}$ at $\mathrm{AT}$ in 7 patients in our study was due to COVID-19. However, a recent cardiac magnetic resonance imaging study revealed that extracellular volume fraction was elevated in participants who recovered from moderate or severe COVID-19 (without cardiac symptoms or structural cardiac abnormalities) compared to healthy controls (26). These results imply that COVID-19 may lead to cardiac damage and subsequent cardiopulmonary insufficiency. Further longitudinal studies need to be conducted to examine this issue.

Additionally, we failed to find any significant differences in the CPET results between patients in the moderate and severe groups. There are two possible reasons for these results. First, as we included only 37 patients, our sample size was relatively small, and a type II error might have occurred. Second, several patients did not cooperate with the examinations, and some patients in the severe group could not tolerate the whole testing, which could have led to bias.

Our study had a few limitations. First, our sample size was small. Further studies should seek to increase the sample size of discharged patients. Second, we did not analyze the laboratory examinations of the patients. Some tests (e.g., C reactive protein, procalcitonin, and interleukins) were done on a small portion of patients at discharge, but these data were not analyzed. Logistic regression analyses could be conducted based on the laboratory tests to identify independent risk 
factors for incomplete radiological resolution at the 6-month follow-up examination. These results could help to predict pulmonary sequelae at an early stage. In our follow-up study, we will enroll more patients with more complete data. Third, the fibrotic-like changes on the follow-up chest CT scans were not pathologically confirmed to be true fibrosis. The follow-up period was too short to assess whether the fibroticlike changes were permanent or reversible. Finally, as we did not do CPET on patients during hospitalization, we could not evaluate any trends in cardiopulmonary changes before and after discharge.

\section{Conclusions}

In conclusion, the most common imaging patterns of COVID-19 survivors 6 months after discharge were interlobular septal thickening, parenchymal band, and traction bronchiectasis. The recovery of the chest CT lesions was slower in patients with severe COVID-19 than those with moderate COVID-19. Chest CT abnormalities resolved without any sequelae in most patients with moderate COVID-19; however, fibrotic-like changes were still present in a considerable proportion of patients with severe COVID-19 at 6 months after discharge. An age $>50$ years old, the severe type of COVID-19, an opacity score at discharge $>4$, and a volume of opacity at discharge $>235 \mathrm{~mL}$ were found to be the main risk factors associated with incomplete radiological resolution. Additionally, lower oxygen uptake at AT may be present in COVID-19 survivors, but there was no significant difference in the CPET results between the moderate and severe groups at 6 months after discharge.

\section{Acknowledgments}

Funding: This work was supported by the Joint Project of Chongqing Science and Technology Commission and Chongqing Public Health Commission (2021MSMX062), the Emergency Research Project of Chongqing Medical University (CQMUNCP0201), and the Wanzhou District COVID-19 Emergency Technology Research Project (wzstc-2020025).

\section{Footnote}

Conflicts of Interest: All authors have completed the ICMJE uniform disclosure form (available at https://dx.doi. org/10.21037/qims-20-1294). The authors have no conflicts of interest to declare.

Etbical Statement: The authors are accountable for all aspects of the work, including ensuring that questions related to the accuracy or integrity of any part of the work have been appropriately investigated and resolved. The study was conducted in accordance with the Declaration of Helsinki (as revised in 2013). This study was approved by the Ethics Committee of Chongqing University Three Gorges Hospital (No: ChiCTR2000029952). Written informed consent was obtained from each individual participant.

Open Access Statement: This is an Open Access article distributed in accordance with the Creative Commons Attribution-NonCommercial-NoDerivs 4.0 International License (CC BY-NC-ND 4.0), which permits the noncommercial replication and distribution of the article with the strict proviso that no changes or edits are made and the original work is properly cited (including links to both the formal publication through the relevant DOI and the license). See: https://creativecommons.org/licenses/by-nc-nd/4.0/.

\section{References}

1. WHO Coronavirus (COVID-19) Dashboard. Available via https://covid19.who.int/

2. Wu J, Wu X, Zeng W, Guo D, Fang Z, Chen L, Huang H, Li C. Chest CT Findings in Patients With Coronavirus Disease 2019 and Its Relationship With Clinical Features. Invest Radiol 2020;55:257-61.

3. Liu M, Zeng W, Wen Y, Zheng Y, Lv F, Xiao K. COVID-19 pneumonia: CT findings of 122 patients and differentiation from influenza pneumonia. Eur Radiol 2020;30:5463-9.

4. Liu M, Song Z, Xiao K. High-Resolution Computed Tomography Manifestations of 5 Pediatric Patients With 2019 Novel Coronavirus. J Comput Assist Tomogr 2020;44:311-3.

5. Xiong Q, Xu M, Li J, Liu Y, Zhang J, Xu Y, Dong W. Clinical sequelae of COVID-19 survivors in Wuhan, China: a single-centre longitudinal study. Clin Microbiol Infect 2021;27:89-95.

6. Fang Y, Zhou J, Ding X, Ling G, Yu S. Pulmonary fibrosis in critical ill patients recovered from COVID-19 pneumonia: Preliminary experience. Am J Emerg Med 2020;38:2134-8.

7. Wei J, Yang H, Lei P, Fan B, Qiu Y, Zeng B, Yu P, Lv J, Jian Y, Wan C. Analysis of thin-section CT in patients with 
coronavirus disease (COVID-19) after hospital discharge. J Xray Sci Technol 2020;28:383-9.

8. Yu M, Liu Y, Xu D, Zhang R, Lan L, Xu H. Prediction of the Development of Pulmonary Fibrosis Using Serial Thin-Section CT and Clinical Features in Patients Discharged after Treatment for COVID-19 Pneumonia. Korean J Radiol 2020;21:746-55.

9. Liu M, Lv F, Huang Y, Xiao K. Follow-Up Study of the Chest CT Characteristics of COVID-19 Survivors Seven Months After Recovery. Frontiers in Medicine 2021;8:636298.

10. Han X, Fan Y, Alwalid O, Li N, Jia X, Yuan M, Li Y, Cao Y, Gu J, Wu H, Shi H. Six-Month Follow-up Chest CT findings after Severe COVID-19 Pneumonia. Radiology 2021;299:E177-86.

11. Du S, Gao S, Huang G, Li S, Chong W, Jia Z, Hou G, Wang YXJ, Zhang L. Chest lesion CT radiological features and quantitative analysis in RT-PCR turned negative and clinical symptoms resolved COVID-19 patients. Quant Imaging Med Surg 2020;10:1307-17.

12. General Office of National Health Committee. Notice on the issuance of a program for the diagnosis and treatment of novel coronavirus (2019-nCoV) infected pneumonia (trial eighth edition). Available online: http://www.nhc.gov. cn/xcs/zhengcwj/202008/0a7bdf12bd4b46e5bd28ca7f9a7f 5e5a/files/a449a3e2e2c94d9a856d5faea2ffof94.pdf

13. Hansell DM, Bankier AA, MacMahon H, McLoud TC, Muller NL, Remy J. Fleischner Society: glossary of terms for thoracic imaging. Radiology 2008;246:697-722.

14. Bruns AH, Oosterheert JJ, El Moussaoui R, Opmeer BC, Hoepelman AI, Prins JM. Pneumonia recovery: discrepancies in perspectives of the radiologist, physician and patient. J Gen Intern Med 2010;25:203-6.

15. Ng CK, Chan JW, Kwan TL, To TS, Chan YH, Ng FY, Mok TY. Six month radiological and physiological outcomes in severe acute respiratory syndrome (SARS) survivors. Thorax 2004;59:889-91.

16. Denina M, Pruccoli G, Scolfaro C, Mignone F, Zoppo M, Giraudo I, Silvestro E, Bertolotti L, Rosati S, Ramenghi U, Garazzino S. Sequelae of COVID-19 in Hospitalized Children: A 4-Months Follow-Up. Pediatr Infect Dis J 2020;39:e458-9.

17. Liu D, Zhang W, Pan F, Li L, Yang L, Zheng D, Wang J, Liang $\mathrm{B}$. The pulmonary sequalae in discharged patients with COVID-19: a short-term observational study. Respir Res 2020;21:125.

18. Antonio GE, Wong KT, Hui DS, Wu A, Lee N, Yuen EH, Leung CB, Rainer TH, Cameron P, Chung
SS, Sung JJ, Ahuja AT. Thin-section CT in patients with severe acute respiratory syndrome following hospital discharge: preliminary experience. Radiology 2003;228:810-5.

19. Zhang D, Zhang C, Li X, Zhao J, An C, Peng C, Wang $\mathrm{L}$. Thin-section computed tomography findings and longitudinal variations of the residual pulmonary sequelae after discharge in patients with COVID-19: a short-term follow-up study. Eur Radiol 2021. [Epub ahead of print]. doi: 10.1007/s00330-021-07799-9.

20. Chang YC, Yu CJ, Chang SC, Galvin JR, Liu HM, Hsiao CH, Kuo PH, Chen KY, Franks TJ, Huang KM, Yang PC. Pulmonary sequelae in convalescent patients after severe acute respiratory syndrome: evaluation with thin-section CT. Radiology 2005;236:1067-75.

21. Chen N, Zhou M, Dong X, Qu J, Gong F, Han Y, Qiu Y, Wang J, Liu Y, Wei Y, Xia J, Yu T, Zhang X, Zhang L. Epidemiological and clinical characteristics of 99 cases of 2019 novel coronavirus pneumonia in Wuhan, China: a descriptive study. Lancet 2020;395:507-13.

22. Zhao YM, Shang YM, Song WB, Li QQ, Xie H, Xu QF, Jia JL, Li LM, Mao HL, Zhou XM, Luo H, Gao YF, Xu AG. Follow-up study of the pulmonary function and related physiological characteristics of COVID-19 survivors three months after recovery. EClinicalMedicine 2020;25:100463.

23. Mezzani A. Cardiopulmonary Exercise Testing: Basics of Methodology and Measurements. Ann Am Thorac Soc 2017;14:S3-11.

24. Weber KT, Janicki JS. Cardiopulmonary exercise testing for evaluation of chronic cardiac failure. Am J Cardiol 1985;55:22A-31A.

25. Huang C, Huang L, Wang Y, Li X, Ren L, Gu X, et al. 6-month consequences of COVID-19 in patients discharged from hospital: a cohort study. Lancet 2021;397:220-32.

26. Li X, Wang H, Zhao R, Wang T, Zhu Y, Qian Y, Liu B, Yu Y, Han Y. Elevated Extracellular Volume Fraction and Reduced Global Longitudinal Strains in Patients Recovered from COVID-19 without Clinical Cardiac Findings. Radiology 2021;299:E230-40.

Cite this article as: Liu M, Lv F, Zheng Y, Xiao K. A prospective cohort study on radiological and physiological outcomes of recovered COVID-19 patients 6 months after discharge. Quant Imaging Med Surg 2021;11(9):4181-4192. doi: 10.21037/qims-20-1294 